\title{
Design of Matched and Mismatched Filters Based on Peak Sidelobe Level Minimization
}

\author{
Mohammad Mehdi PISHROW ${ }^{1}$, Jamshid ABOUEI ${ }^{1}$, Hesam GHAFERI $^{2}$ \\ ${ }^{1}$ WINEL Research Laboratory, Dept. of Electrical Engineering, Yazd University, Yazd, Iran \\ ${ }^{2}$ Dept. of Electrical Engineering, University of Applied Science and Technology (UAST), ITMC Branch, Shiraz, Iran \\ pishrow.m@gmail.com, abouei@yazd.ac.ir, hesam.ghaferi@gmail.com
}

Submitted October 7, 2020 / Accepted April 19, 2021

\begin{abstract}
This paper focuses on the design of matched filters with low peak sidelobe level as well as mismatched filters with low loss in processing gain and peak sidelobe level, for phase codes. We propose an algorithm which employs the least- $p^{\text {th }}$ norm minimax based on the genetic algorithm, and a method based on the semidefinite programming to deal respectively with the resulting matched and mismatched optimization problems. A framework is also presented to design mismatched filters that are robust to Doppler shifts. Simulation results show that using the proposed methods for finding matched filters leads to better peak sidelobe level and integrated sidelobe level for binary and polyphase codes compared to previous works. In addition, the mismatched filters designed by the proposed methods have very low peak sidelobe level in the binary and polyphase cases.
\end{abstract}

\section{Keywords}

Matched filter, mismatched filter, peak sidelobe level, loss in processing gain, Doppler robustness

\section{Introduction}

The matched filter is the optimal filter to detect a single target in the Gaussian noise such that the Signal-to-Noise Ratio (SNR) at the reception side is maximized [1]. Although matched filters benefit from the maximal processing gain, they suffer from the relatively high autocorrelation function sidelobes that impresses the detection of multi-target in which weak targets are masked in the sidelobes of stronger targets or a strong clutter. This problem appears in many applications such as classic radar range/Doppler ambiguity functions [2]. Based on the waveform used at the transmitter, such as linear chirp signals and particularly phase codes, some sidelobe reduction techniques are used at the receiver. One possible solution to suppress the sidelobe level for linear chirp signals is to apply a weighting window such as Hamming at the reception [3], which imposes some resolution loss due to the mainlobe broadening and some loss in the processing gain.
For phase codes such as Binary Phase Shift Keying (BPSK), weighting windows cannot be applied, however, it is possible to find optimal filters that optimize the Peak Sidelobe Ratio (PSLR) parameter [4]. The waveform design in transmit signals and received filters is one of the most important issues in radar systems. There exist lots of research to find optimal filters based on various criteria [5-8]. The first study in the generation of filters for the sidelobe reduction was framed in [9] where the main goal is to minimize the sidelobe energy over multiple input sequences.

The non-dominated sorting genetic algorithm-II is used in [10] to generate biphase pulse compression codes from length 49 to 100 taking PSL and ISL as the objective functions. In [11], an efficient method is presented to reduce the computational complexity of nearest-neighbor search-based optimization algorithms of a phase coded waveform. A mismatched filter design for MIMO radars is proposed in [12] that minimizes the interference and jamming power at the filter output, while the peak sidelobe and cross-correlation levels for all Doppler frequencies are constrained to desired values. This method employs the semidefinite relaxation to obtain a convex optimization problem. The authors in [13] show that gradients for a large variety of cost functions can be computed very efficiently in the gradient descent-based approach compared to sequence design. The authors in [7] propose two schemes to further suppress sidelobe levels in distributed MIMO radars, i.e., orthogonal phase-coded waveforms with an expanded mainlobe and its mismatched filters design. New sequence set design methods with accurately controlled autocorrelation and cross-correlation sidelobe levels were developed in [14] for the design of unimodular sequences with accurately controlled correlation properties within the lag interval of interest. The concept of majorization-minimization in combination with the second order cone programming and semidefinite programming was used in [15] to design waveforms for Time-DivisionMultiplexing (TDM) MIMO radar systems. Using a different approach, a frequency domain expression was presented in [16] for the waveform autocorrelation. Then, using the proximal multiplier process, minimum local PSL waveforms were designed with correlation and spectral constraints. Note 
that the mismatched filter design for a particular transmitted code is a convex optimization problem, while the matched filter design with unit modulus constraint is a non-convex problem. According to these concepts, the attention of researchers has been devoted to proposing efficient algorithms to solve optimization problems in designing matched and mismatched filters. Note that in most literature, the key emphasis is on the design of low ISL-based codes due to the complexities occur in the design of PSL-based codes. Motivated by the above considerations and to design the matched filters with low PSL, we transform the matched filter problem into an optimization problem and propose an algorithm that uses the least- $\mathrm{p}^{\text {th }}$ norm minimax based on the genetic algorithm to solve it. An optimization problem is constructed to design the mismatched filters and solved through exploiting the semidefinite programming which uses efficient interior point methods. Toward this goal, we consider the following criteria: $i$ ) minimization of PSL, and $i$ ) minimization of Loss in Processing Gain (LPG). Simulation results show that using the proposed method for finding matched filters results in better PSL and Integrated Sidelobe Level (ISL) for polyphase codes compared to previous works. In addition, the mismatched filters designed by the proposed method have the lowest PSL.

The rest of the paper is organized as follows. In Sec. 2, we present the matched and mismatched filter design procedure with the mentioned criteria and the mismatched filter with the Doppler constraints by solving optimization problems. In Sec. 3, comprehensive simulation results are provided. In Sec. 4, an overview of the results and some conclusion remarks are presented.

\section{Signal Model}

Matched Filter Design: Consider an active sensing system which transmits a complex pulse coded waveform $\mathbf{s}=\left[s_{0}, s_{1}, \ldots, s_{L-1}\right]^{\mathrm{T}}$ with $s_{i}=\exp \left(\mathrm{j} \varphi_{i}\right)$, where $\varphi_{i}$ denotes the phase of $i^{\text {th }}$ transmitted pulse coded waveform $s_{i}$ and $L$ indicates the transmitted waveform code length. The corresponding matched filter coefficients are defined as $\tilde{\mathbf{s}}=\left[s_{L-1}, s_{L-2}, \ldots, s_{0}\right]^{\mathrm{H}}$ and the output result of matched filtering is obtained as $\mathbf{r}=\mathbf{s} \otimes \tilde{\mathbf{s}}$. To facilitate our analysis, we reform the convolution operation $\otimes$ into the matrix multiplication form in which $q^{\text {th }}$ element of $\mathbf{r}$ can be defined as $r_{q}=\mathbf{s}^{\mathrm{H}} \mathbf{J}_{q} \mathbf{s}$, where the element $r_{q}, \forall q \neq 0$, indicates the range sidelobes, $r_{0}$ denotes the mainlobe, and $\mathbf{J}_{q}$ is the shift matrix defined by [17]

$$
\mathbf{J}_{q}=\left[\begin{array}{cc}
\mathbf{0}_{(L-q) \times q} & \mathbf{I}_{(L-q) \times(L-q)} \\
\mathbf{0}_{q \times q} & \mathbf{0}_{q \times(L-q)}
\end{array}\right]_{L \times L}, 0 \leqslant q \leqslant L .
$$

Note that $\mathbf{J}_{q}=\mathbf{J}_{-q}^{\mathrm{H}}$ and $r_{-q}=\mathbf{s}^{\mathrm{H}} \mathbf{J}_{-q} \mathbf{s}=\left(\mathbf{s}^{\mathrm{H}} \mathbf{J}_{q} \mathbf{s}\right)^{\mathrm{H}}=r_{q}^{*}$, i.e., the sidelobes $r_{q}$ have the conjugate symmetric relationship; then, $\left|r_{q}\right|=\left|r_{-q}\right|$. Hence, for the pulse coded waveform $\mathbf{s}$, the PSL of the matched filter output is defined as $\max _{q}\left|r_{q}\right|, 1 \leqslant q \leqslant L-1$. To suppress the PSL, we should solve the following optimization problem

$$
\min _{\Phi} \max _{q}\left|r_{q}(\Phi)\right|, 1 \leqslant q \leqslant L-1
$$

where $\Phi=\left[\varphi_{1}, \ldots, \varphi_{L-1}\right]$ is the phase vector of pulse coded waveform s. Recalling that $\left|s_{\mathrm{k}}\right|=\left|\mathrm{e}^{\mathrm{j} \varphi_{\mathrm{k}}}\right|=1, k \in[1, L-1]$, the optimization problem (2) is non-convex. To solve this problem, we propose an algorithm that uses the Least- ${ }^{\text {th }}$ norm Minimax based on the Genetic Algorithm (LMGA). In order to apply the LMGA to the problem, it is necessary to transform (2) into a norm form. Stacking all the sidelobes absolute values of $\mathbf{r}$ into a vector, the cost function (2) is rewritten as $\min _{\Phi} f_{p \rightarrow \infty}(\Phi)$, where $f_{p}(\Phi)=$ $\left\|\left[\left|r_{1}(\Phi)\right|, \ldots,\left|r_{L-1}(\Phi)\right|\right]\right\|_{p}$. Note that the minimum value of $\left\|\left[\left|r_{1}\right|, \ldots,\left|r_{L-1}\right|\right]\right\|_{\infty}$ can be achieved through increasing the power $p$. The main goal of utilizing LMGA is to find codes such that their matched filters' output have as low PSL as possible. The proposed LMGA includes the initialization, phase selection, new phases generation, and stopping criteria steps described in Algorithm 1.

The output of the algorithm consists of the best population of chromosomes, i.e., $\mathbf{D}$ which denotes the phase vector by which the transmitted phase code is constructed and the corresponding fitness function value $\mathbf{f}_{\mathrm{i}}$, i.e., the sidelobe level value of the corresponding matched filter. In summary, the pseudocode of the LMGA is given in Algorithm 1 and its glossary is illustrated in Tab. 1.

Mismatched Filter Design: Although a matched filter is optimal at the receiver in the case of the additive white Gaussian noise, using a mismatched filter may cause a further PSL suppression at the cost of the LPG. Consider an exactly known pulse coded waveform $\mathbf{s}=\left[s_{0}, s_{1}, \ldots, s_{L-1}\right]^{\mathrm{T}}$ obtained from the LMGA. Let the corresponding mismatched filter coefficients of $\mathbf{s}$ be denoted by $\mathbf{h}=\left[h_{0}, h_{1}, \ldots, h_{M-1}\right]^{\mathrm{T}}$, in which $M \geqslant L$ is the mismatched filter length. The output of the mismatched filter is obtained by $\boldsymbol{\Omega}=\mathbf{s} \otimes \mathbf{h}$. For the ease of our analysis, we choose both $L$ and $M$ to be even (or odd) numbers and construct the pulse coded waveform $\hat{\mathbf{s}}$ as $\hat{\mathbf{s}}_{M \times 1}=\left[\mathbf{0}_{1 \times(M-L) / 2}, \mathbf{s}^{\mathrm{T}}{ }_{1 \times L}, \mathbf{0}_{1 \times(M-L) / 2}\right]^{\mathrm{T}}$, where we added $(M-L) / 2$ zeros at the beginning and at the end of $\mathbf{s}$. To reform the convolution structure of $\boldsymbol{\Omega}$, we define a complex matrix $\mathbf{K}$ with dimension $(2 M-1) \times M$ as

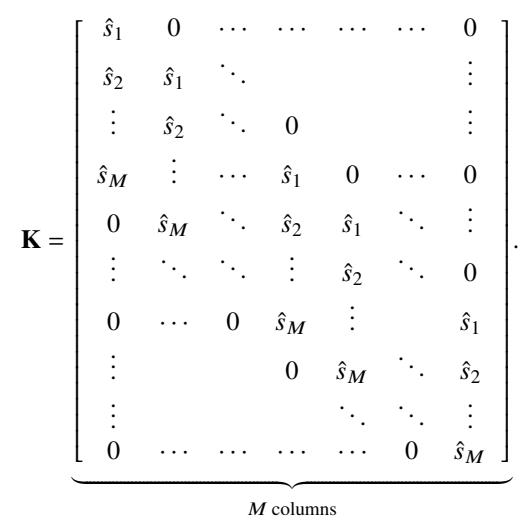


Hence, the output of the mismatched filter can be defined by the following matrix multiplication

$$
\mathbf{\Omega}=\mathbf{s} \otimes \mathbf{h}=\mathbf{K h}=\left[\omega_{-M}, \omega_{-M+1}, \ldots, \omega_{-1}, \omega_{0}, \omega_{1}, \ldots, \omega_{M}\right]^{\mathrm{T}}
$$

where $\omega_{0}$ denotes the mainlobe, and the range sidelobes of $\boldsymbol{\Omega}$ can be expressed as

$$
\mathbf{U}=\left[\omega_{-M}, \omega_{-M+1}, \ldots, \omega_{-1}, \omega_{1}, \ldots, \omega_{M}\right]^{\mathrm{T}} .
$$

The following measures can be used to design and evaluate the performance of mismatched filters.

PSL: The PSL of a mismatched filter's output is used to evaluate the range sidelobe level and can be defined as

$$
\mathrm{PSL}=\|\mathbf{U}\|_{\infty}=\max _{j}\left|\omega_{j}\right|,-M \leqslant j \leqslant M, j \neq 0 .
$$

PSLR: This parameter computes the ratio between the maximum sidelobe level and the mainlobe and is defined as

$$
\operatorname{PSLR}=\frac{\max _{j}\left|\omega_{j}\right|}{\omega_{0}},-M \leqslant j \leqslant M, j \neq 0 .
$$

LPG: Any mismatched filter results in an LPG expressed as the ratio between the SNR of mismatched filter $\mathrm{SNR}_{\mathrm{MM}}$, and the optimal SNR at the output of matched filter $\mathrm{SNR}_{\mathrm{M}}$. Given a pulse coded waveform $\mathbf{s}$, and its corresponding mismatched filter $\mathbf{h}$, in the case of zero mean white Gaussian noise with variance $\sigma^{2}$, the LPG is defined as

$$
\begin{aligned}
\mathrm{LPG} & =10 \log _{10}\left(\frac{\mathrm{SNR}_{\mathrm{MM}}}{\mathrm{SNR}_{\mathrm{M}}}\right)=10 \log _{10}\left(\frac{\left(\mathbf{h}^{\mathrm{H}} \mathbf{s}\right)\left(\mathbf{h}^{\mathrm{H}} \mathbf{s}\right)^{\mathrm{H}} / \sigma^{2}\left(\mathbf{h}^{\mathrm{H}} \mathbf{h}\right)}{\left(\mathbf{s}^{\mathrm{H}} \mathbf{s}\right)\left(\mathbf{s}^{\mathrm{H}} \mathbf{s}\right)^{\mathrm{H}} / \sigma^{2}\left(\mathbf{s}^{\mathrm{H}} \mathbf{s}\right)}\right) \\
& =10 \log _{10}\left(\frac{\left|\mathbf{h}^{\mathrm{H}} \mathbf{s}\right|^{2}}{\left(\mathbf{h}^{\mathrm{H}} \mathbf{h}\right)\left(\mathbf{s}^{\mathrm{H}} \mathbf{s}\right)}\right) .
\end{aligned}
$$

It is worth mentioning that the SNR at the output of the matched filter with unit absolute values and mismatched filters considering the additive zero-mean white Gaussian noise with unit variance can be respectively simplified to $\mathrm{SNR}_{\mathrm{M}}=10 \times \log _{10}(L)$ and $\mathrm{SNR}_{\mathrm{MM}, \mathrm{dB}}=\mathrm{SNR}_{\mathrm{M}, \mathrm{dB}}-|\mathrm{LPG}|$.

ISLR: This parameter obtains the ratio between the accumulation of the sidelobes and the peak level for both matched and mismatched filters and can be defined as ISLR $=\frac{\mathbf{U}^{\mathrm{H}} \mathbf{U}}{\left|\omega_{0}\right|^{2}}=\frac{\|\mathbf{U}\|^{2}}{\left|\omega_{0}\right|^{2}}$. The closed form of the optimal mismatched filter that minimizes the ISLR can be expressed as

$$
\mathbf{h}_{\text {ISLR_opt }}=\frac{\left(\mathbf{s}^{\mathrm{H}} \mathbf{s}\right)\left(\mathbf{U} \mathbf{U}^{\mathrm{H}}\right)^{-1} \mathbf{s}}{\mathbf{s}^{\mathrm{H}}\left(\mathbf{U} \mathbf{U}^{\mathrm{H}}\right)^{-1} \mathbf{s}} .
$$

While (9) is the optimal filter based on ISLR, it is not optimal for the PSL case. This motivates us to define a PSLbased mismatched filter optimization problem as follows

$$
\begin{aligned}
& \min _{\mathbf{h}}\|\mathbf{U}\|_{\infty} \\
\text { s.t. } & \mathbf{s}^{\mathrm{H}} \mathbf{h}=\mathbf{s}^{\mathrm{H}_{\mathbf{S}}},
\end{aligned}
$$

\begin{tabular}{|l|l|}
\hline cop & Crossover point selected randomly \\
$\mathbf{D}$ & Population \\
$\mathbf{D}^{A}, \mathbf{D}^{B}$ & Row numbers selected for crossover operation \\
$m_{\text {col }}$ & Chromosome component numbers to be mutated \\
$m_{\text {row }}$ & Chromosome numbers to be mutated \\
$N_{\text {kep }}$ & Number of chromosomes kept \\
$N_{\text {mating }}$ & Number of pairing operations \\
$N_{\text {mut }}$ & Number of mutation operations \\
$N_{\text {pop }}$ & Population size \\
$\mathbf{P}$ & Selection probability of chromosomes \\
$p$ & Norm power \\
$\mathbf{P}_{\mathrm{c}}$ & Cumulative probability of selection process \\
$r_{\mathrm{m}}$ & Mutation rate \\
rnd $_{1}$, rnd \\
$\mathbf{V}_{2}$ & Random vectors of size $1 \times N_{\text {mating }}$ \\
$\mathbf{V}_{\text {cop-inv }}$ & A vector filled with numbers 1 to $c o p$ \\
$\mathbf{V}_{\mathrm{k}}$ & A vector filled with numbers $c o p+1$ to $L$ \\
$\mathbf{V}_{\mathrm{k} \text {, odd }}$ & A vector filled with numbers 1 to $N_{\mathrm{k}}$ \\
$\mathbf{V}_{\mathrm{k} \text {, even }}$ & Odd numbers of $\mathbf{V}_{k}$ \\
\hline
\end{tabular}

Tab. 1. Glossary of Algorithm 1

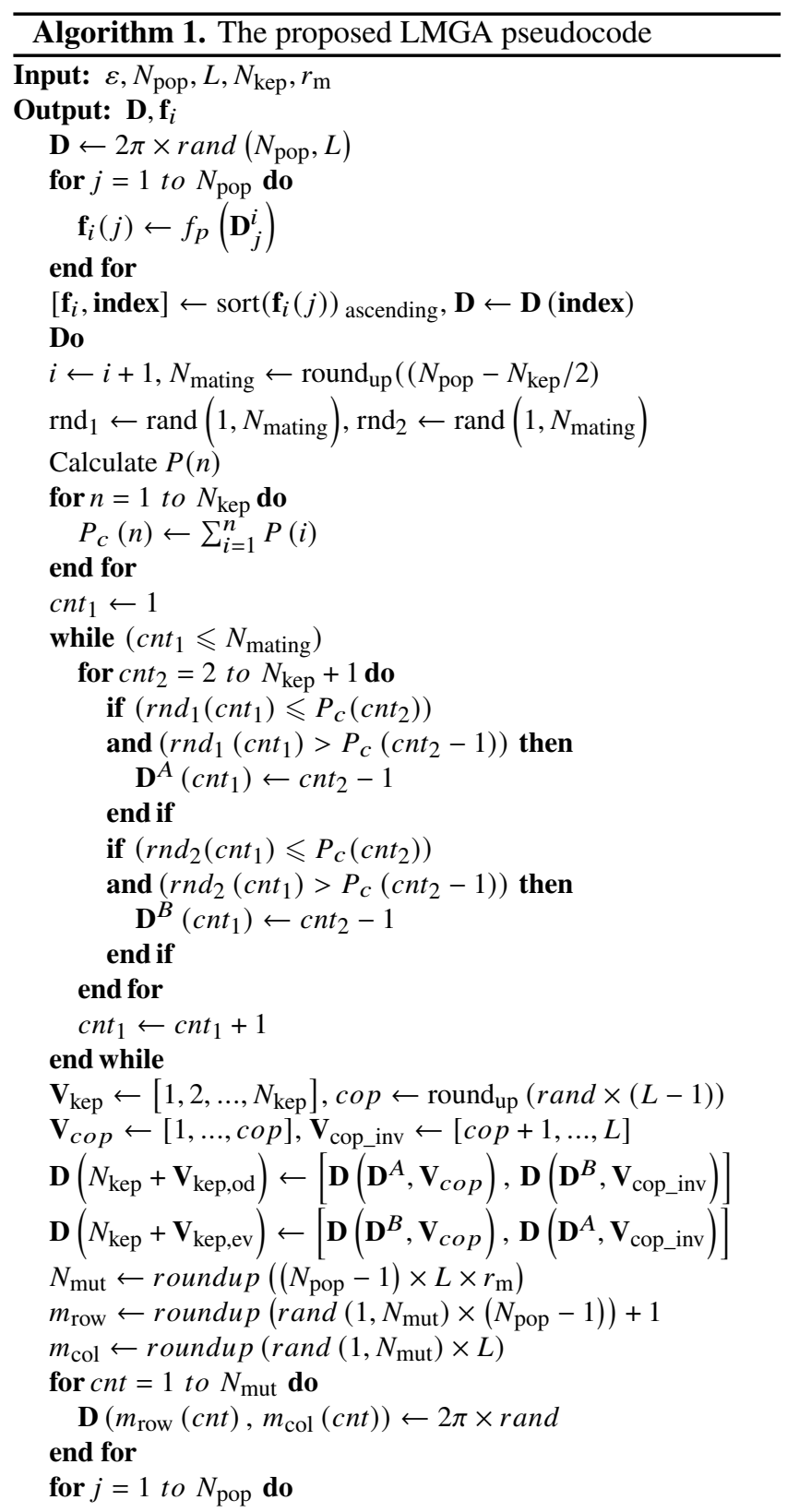




$$
\begin{aligned}
& \quad f_{i}(j) \leftarrow f_{p}\left(\mathbf{D}_{j}^{i}\right) \\
& \text { end for } \\
& {\left[\mathbf{f}_{i}, \text { index }\right] \leftarrow \operatorname{sort}\left(\mathbf{f}_{i}(j)\right) \text { ascending }, \mathbf{D} \leftarrow \mathbf{D}(\text { index }), p=2^{i+1}} \\
& \text { while }\left|f_{i}(1)-f_{i-1}(1)\right|>\varepsilon
\end{aligned}
$$

where the constraint $\mathbf{s}^{\mathrm{H}} \mathbf{h}=\mathbf{s}^{\mathrm{H}} \mathbf{s}$ is included to forsake the null solution. The objective function of (10) is convex because every norm of vectors is convex [18]. In addition, the constraint of (10) is linear, therefore it is also a convex function. Thus, optimization problem (10) is convex and has an optimal solution [18]. The interesting feature of such a convex formulation is that it allows to easily include additional constraints, as long as they remain convex. For example, it is possible to add an LPG constraint to control the loss induced by the mismatched filter. Replacing the constraint $\mathbf{s}^{\mathrm{H}} \mathbf{h}=\mathbf{s}^{\mathrm{H}} \mathbf{s}$ in the LPG formula, the LPG is simplified as $10 \times \log _{10}\left(\mathbf{s}^{\mathrm{H}} \mathbf{s} / \mathbf{h}^{\mathrm{H}} \mathbf{h}\right)$. In addition, the LPG constraint can be defined as LPG $\geqslant-10 \times \log _{10}\left(\delta_{1}\right)$, where $\delta_{1} \geqslant 1$ is a predefined free variable to control the LPG. The above inequality results in $\mathbf{h}^{\mathrm{H}} \mathbf{h} \leqslant \delta_{1} \mathbf{s}^{\mathrm{H}} \mathbf{s}$, which is quadratic convex because $\delta_{1} \mathbf{s}^{\mathrm{H}} \mathbf{s}$ is constant. The LPG constraint is of primary importance, since it permits to ensure that the good PSLR provided by the optimal filter $\mathbf{h}$ is not achieved at the cost of a large LPG, or conversely, it is possible to limit the LPG to some reasonable predefined loss. Now we can define the PSL problem of a mismatched filter with the LPG constraint as follows and ensure that it is also a convex problem

$$
\begin{array}{ll}
\min _{\mathbf{h}}\|\mathbf{U}\|_{\infty} \\
\text { s.t. } & \left\{\begin{array}{l}
\mathbf{s}^{\mathrm{H}} \mathbf{h}=\mathbf{s}^{\mathrm{H}} \mathbf{s} \\
\mathbf{h}^{\mathrm{H}} \mathbf{h} \leqslant \delta_{1} \mathbf{s}^{\mathrm{H}} \mathbf{s}
\end{array}\right.
\end{array}
$$

To solve this problem, we have applied Semidefinite Programming (SDP) approach. Since the optimization problem is convex, SDP would approach to the optimal solution, however, one needs to compare its computational complexity with other methods. Many literature present solutions based on SDP which use efficient interior point methods for solving convex problems [18]. Various softwares have been developed for solving convex optimization problems. We use YALMIP (a MATLAB package [19]) to solve the convex program of mismatched filter design. In addition, we apply a gradientbased approach [11], which is an effective scheme in finding binary and polyphase sequences. The optimal mismatched filter is found in both cases due to the convexity of the problem, however as shown in Tab. 2, the computational complexities, or equivalently, the run time of algorithms are different. Note that in the case of short code lengths, the run times are almost the same, but in longer code lengths, the run times are different. As an example, the run times of a BPSK code with length 100 for different schemes achieved by the LMGA, are reported in Tab. 2. In addition, PSLR, LPG and

\begin{tabular}{|c|c|c|c|c|c|c|c|}
\hline Code & $L$ & M & PSLR & LPG & ISLR & $\begin{array}{l}\text { RT of proposed } \\
\text { alg. [s] }\end{array}$ & RT of [11] [s] \\
\hline \multirow{5}{*}{$\begin{array}{l}\frac{u}{v} \\
\frac{n}{n}\end{array}$} & \multirow{5}{*}{$\stackrel{8}{\circ}$} & 150 & $\overline{-31.56}$ & $\overline{-0.73}$ & $\begin{array}{l}-8.92 \\
\end{array}$ & $\overline{2.03}$ & $\overline{2.43}$ \\
\hline & & 200 & -34.38 & -0.80 & -10.92 & 2.23 & 2.71 \\
\hline & & 300 & -41.63 & -0.89 & -16.45 & 3.37 & 5.17 \\
\hline & & 500 & -48.87 & -1.13 & -21.69 & 8.11 & 15.30 \\
\hline & & 1000 & -56.34 & -1.32 & -26.25 & 14.92 & 37.81 \\
\hline
\end{tabular}
ISLR metrics for the corresponding mismatched filters are given in Tab. 2. It is observed from this table that the proposed approach based on semidefinite programming using YALMIP, has a lower run time than that of [11].

Tab. 2. PSLR, LPG, ISLR, and Run Times (RT) of the proposed algorithm using SDP and formulation of [11].
Mismatched Filter Design with Doppler Sidelobe Suppression: In practical applications, it is desirable that the pulse compression output be robust to the Doppler effect. The moving targets in active sensing result in such Doppler shifts and the output of the correlator filters should take into account this Doppler effect, so the mentioned targets can be detected and resolved correctly. According to the Doppler effect in the active sensing applications, it is necessary to design a mismatched filter with the Doppler effect taking into account. We use superscript "d" to show the Doppler effect. Consider a pulse coded waveform $\mathbf{s}=\left[s_{0}, s_{1}, \ldots, s_{L-1}\right]^{\mathrm{T}}$ obtained from the LMGA with its corresponding mismatched filter coefficients $\mathbf{c}=\left[c_{0}, c_{1}, \ldots, c_{M-1}\right]^{\mathrm{T}}$. The received Doppler modulated waveform may be defined as $\mathbf{s}^{\mathrm{d}}=\left[s_{0}, s_{1} e^{\mathrm{j} 2 \pi f_{\mathrm{d}}}, \ldots, s_{L-1} e^{\mathrm{j} 2 \pi(L-1) f_{\mathrm{d}}}\right]^{\mathrm{T}}$ where $f_{\mathrm{d}}$ is the Doppler frequency of the target. In this case, the mismatched filter output is obtained by $\boldsymbol{\Omega}^{\mathrm{d}}=\mathbf{s}^{\mathrm{d}} \otimes \mathbf{c}$ where its sidelobes based on (4) is given by $\mathbf{U}^{\mathrm{d}}=\left[\omega_{-M}^{\mathrm{d}}, \omega_{-M+1}^{\mathrm{d}}, \ldots, \omega_{-1}^{\mathrm{d}}, \omega_{1}^{\mathrm{d}}, \ldots, \omega_{M}^{\mathrm{d}}\right]^{\mathrm{T}}$. The filter should be designed such that the PSL is minimum in a specified region of Doppler frequencies $\mathcal{R}=\left[-f_{\mathrm{d}_{\max }}, f_{\mathrm{d}_{\max }}\right]$. The PSL of mismatched filter output $\mathbf{\Omega}^{\mathrm{d}}$ with $f_{\mathrm{d}} \in \mathcal{R}$ is defined as

$$
\operatorname{PSL}^{\mathrm{d}}=\left\|\mathbf{U}^{\mathrm{d}}\right\|_{\infty}=\max _{j}\left|\mathbf{U}_{j}^{\mathrm{d}}\right|,-M \leqslant j \leqslant M, j \neq 0 .
$$

Therefore, the problem of mismatched filter design with the Doppler sidelobe suppression considering the LPG constraint can be written as

$$
\begin{array}{ll}
\min _{\mathbf{c}}\left\|\mathbf{U}^{\mathrm{d}}\right\|_{\infty} \\
\text { s.t. } & \left\{\begin{array}{l}
\mathbf{s}^{\mathrm{H}} \mathbf{c}=\mathbf{s}^{\mathrm{H}} \mathbf{s} \\
\mathbf{c}^{\mathrm{H}} \mathbf{c} \leqslant \delta_{2} \mathbf{s}^{\mathrm{H}} \mathbf{s}
\end{array}\right.
\end{array}
$$

where $\delta_{2} \geqslant 1$ is a predefined variable to determine the LPG range and is set manually. It should be noted that we discretize the Doppler region and consider all the corresponding different Doppler values to cover the whole desired Doppler region. It is shown that the above mismatched filter design with the Doppler sidelobe suppression is a convex optimization problem, in the same way that the convexity of mismatched filter design has been proved.

\section{Numerical Results}

In this section, numerical simulation results are presented to evaluate the performance of Algorithm 1 for the matched filter design. In addition, the performance of the mismatched filter design using the proposed algorithms in Sec. 2 is analyzed. Finally, the performance of the designed mismatched filter in Sec. 2 considering the Doppler effect is examined through the simulations.

Results of Matched Filter Design: In the first part of our simulations, we consider two cases of discrete phase and continuous phase (polyphase) to design matched codes using the LMGA. In the discrete phase case, BPSK codes with $\varphi_{i} \in\{0, \pi\}$ are considered to report in Tab. 3. To show the performance of the proposed method, the corresponding results of the BPSK and polyphase codes using the LMGA for some values of $L$ are compared respectively with BPSK codes found by 1bCAN [20] and $i$ ) polyphase Barker codes, and $i$ ) polyphase codes found by Cyclic Algorithm-New (CAN) [21] with random and Golomb codes as its initial values in Tab. 3. Note that the BPSK codes found by the LMGA achieve the globally best peak sidelobe levels for those code lengths. As we can see in Tab. 3, the polyphase codes found by the LMGA is overally better than the codes found by CAN and are near to polyphase Barker codes in the sense of PSLR and ISLR. 


\begin{tabular}{|c|c|c|c|c|c|c|c|c|c|c|c|c|}
\hline \multirow{2}{*}{$\mathrm{L}$} & \multicolumn{2}{|c|}{ BPSK (LMGA) } & \multicolumn{2}{|c|}{ BPSK (1bCAN) } & \multicolumn{2}{|c|}{ Polyphase (LMGA) } & \multicolumn{2}{|c|}{ Polyphase Barker Code } & \multicolumn{2}{|c|}{ CAN (Random) } & \multicolumn{2}{|c|}{ CAN (Golomb) } \\
\hline & PSLR(dB) & ISLR(dB) & PSLR(dB) & ISLR(dB) & PSLR(dB) & ISLR(dB) & PSLR(dB) & ISLR(dB) & PSLR(dB) & ISLR(dB) & PSLR(dB) & ISLR(dB) \\
\hline 14 & -16.90 & -7.12 & -16.90 & -10.13 & -22.72 & -9.24 & -22.92 & -9.29 & -21.45 & -12.38 & -20.46 & -11.22 \\
\hline 15 & -17.50 & -6.89 & -13.98 & -11.76 & -23.52 & -11.89 & -23.52 & -11.62 & -15.56 & -7.68 & -18.87 & -11.29 \\
\hline 16 & -18.06 & -6.60 & -12.04 & -9.03 & -24.08 & -10.47 & -24.08 & -10.55 & -18.25 & -7.85 & -20.68 & -12.37 \\
\hline 17 & -18.59 & -6.55 & -18.59 & -9.56 & -24.61 & -12.76 & -24.61 & -12.57 & -21.83 & -12.26 & -19.19 & -12.27 \\
\hline 18 & -19.08 & -8.10 & -19.08 & -11.13 & -25.10 & -11.84 & -25.10 & -11.86 & -21.53 & -11.88 & -22.44 & -11.36 \\
\hline 19 & -19.55 & -6.88 & -16.03 & -9.45 & -25.38 & -11.43 & -25.57 & -11.53 & -19.90 & -9.53 & -17.76 & -8.24 \\
\hline 20 & -20 & -7.20 & -16.48 & -11.87 & -25.57 & -11.27 & -25.57 & -11.53 & -22.85 & -11.54 & -20.76 & -11.17 \\
\hline 21 & -20.42 & -8.11 & -16.90 & -9.45 & -26 & -11.67 & -26.44 & -11.59 & -19.38 & -9.25 & -19.32 & -10.35 \\
\hline 22 & -17.31 & -7.93 & -17.31 & -10.94 & -26.06 & -12.29 & -26.85 & -12.33 & -23.31 & -11.95 & -22.17 & -12.39 \\
\hline 23 & -17.69 & -7.50 & -17.69 & -9.53 & -26.72 & -12.88 & -27.23 & -13.04 & -21.52 & -9.95 & -22.96 & -14.47 \\
\hline 24 & -18.06 & -9.03 & -15.56 & -9.54 & -27 & -11.58 & -27.60 & -11.63 & -21.92 & -11.61 & -22.13 & -12.61 \\
\hline 25 & -21.94 & -8.50 & -15.92 & -8.72 & -27.51 & -12.09 & -27.96 & -12.20 & -21.42 & -11.19 & -21.76 & -13.98 \\
\hline 26 & -18.76 & -8.76 & -18.76 & -9.91 & -27.81 & -13.13 & -28.30 & -13.40 & -19.75 & -10.21 & -22.40 & -12.74 \\
\hline 27 & -19.08 & -9.94 & -19.08 & -12.94 & -27.94 & -12.57 & -28.63 & -12.51 & -20.22 & -10.85 & -19.91 & -11.30 \\
\hline 28 & -22.92 & -8.94 & -19.40 & -9.80 & -28.28 & -12.51 & -28.94 & -12.48 & -19.25 & -10.48 & -19.26 & -11.96 \\
\hline 29 & -19.70 & -8.31 & -17.21 & -9.71 & -29.06 & -14.16 & -29.25 & -14.05 & -21.03 & -9.11 & -20.50 & -11.63 \\
\hline 30 & -20 & -8.82 & -17.50 & -9.95 & -29.05 & -12.80 & -29.54 & -12.75 & -22.00 & -10.48 & -25.09 & -12.49 \\
\hline 31 & -20.28 & -8.55 & -17.79 & -10.43 & -29.47 & -13.43 & -29.83 & -13.43 & -21.13 & -10.27 & -21.16 & -9.67 \\
\hline 40 & -22.50 & -8.70 & -18.06 & -9.12 & -31.73 & -13.43 & -32.04 & -14.72 & -20.39 & -10.71 & -27.21 & -14.13 \\
\hline 70 & -24.86 & -7.80 & -21.34 & -9.13 & -35.73 & -16.38 & -36.90 & -16.34 & -23.58 & -11.44 & -26.77 & -14.44 \\
\hline
\end{tabular}

Tab. 3. Results of matched filter design: PSLR and ISLR metrics for BPSK and polyphase codes found by the LMGA compared with 1bCAN [20], polyphase Barker codes, and CAN [21].

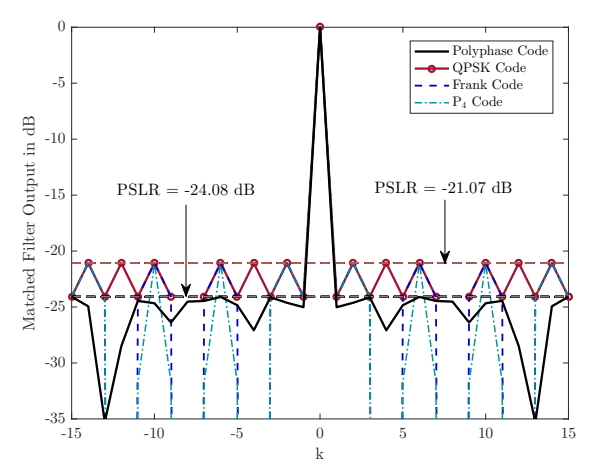

Fig. 1. Matched filter outputs of designed polyphase and QPSK codes, Frank code and $\mathrm{P}_{4}$ code.

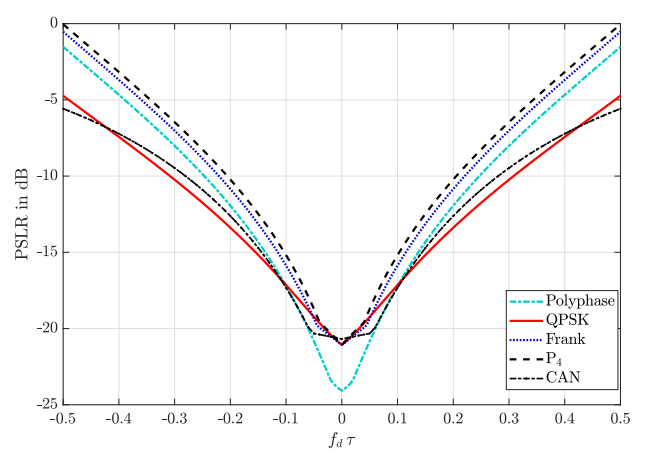

Fig. 2. PSLR versus Doppler shift for the designed polyphase, QPSK, CAN, Frank and $\mathrm{P}_{4}$ codes.

The matched filter outputs of QPSK and polyphse codes with $L=16$, found by the LMGA, are compared with the $\mathrm{P}_{4}$ code and Frank code [4] in Fig. 1. The curves show that the PSLR of the designed polyphase code is lower than that of Frank and $\mathrm{P}_{4}$ codes, while the PSLR of the designed QPSK code is the same as the $\mathrm{P}_{4}$ and Frank codes. This is due to the fact that the phases of the designed polyphase are continuous, while the $\mathrm{P}_{4}$ and Frank phases are constant during the matched filter design. In addition, based on the results in Tab. 3, the ISLR parameter for the designed polyphase code is equal to $-10.47 \mathrm{~dB}$, which is lower than that of $\mathrm{P}_{4}$ code with ISLR of $-10.04 \mathrm{~dB}$, Frank code with ISLR of $-9.03 \mathrm{~dB}$ and de- signed QPSK with ISLR of $-8.06 \mathrm{~dB}$. We consider a typical active sensing system with the carrier frequency $f_{\mathrm{c}}=3 \mathrm{GHz}$, bandwidth $B=2 \mathrm{MHz}$ and the maximum target velocity $v_{\max }=1000 \mathrm{~m} / \mathrm{s}$. The PSLR versus Doppler shift for the designed polyphase and QPSK codes, CAN, $\mathrm{P}_{4}$ and Frank codes are shown in Fig. 2. Clearly, in the plotted Doppler region, the designed polyphase and QPSK matched codes as well as CAN are less sensitive to the Doppler frequency than $\mathrm{P}_{4}$ and Frank codes. Otherwise stated, the mainlobe of Frank and $\mathrm{P}_{4}$ codes are masked by the sidelobes faster than CAN and the designed polyphase and QPSK matched codes.

Among the designed matched codes, we choose one code in each type of BPSK and polyphase codes to design the corresponding mismatched filters. The BPSK code with $L=28$ and the polyphase code with $L=30$ are selected from Tab. 3. The matched filter outputs of the selected codes are plotted in Fig. 3, where we see that the PSL of the chosen BPSK and polyphase codes are equal to 2 and 1.05 , respectively.

Results of Mismatched Filter Design: Given the complexity of the waveform optimization for active sensing applications, it is rational to apply mismatched filters in the receivers to enhance the performance of the filter's output. In this regard, we optimize mismatched filters for a set of BPSK with $L=28$, polyphase waveforms with $L=30$, and different number of filter coefficients at the receiver. The mismatched filters are designed to have as low PSL as possible, while the LPG magnitude is constrained to be lower than $0.8 \mathrm{~dB}$ for BPSK and polyphase codes. The results of mismatched filter optimization for BPSK and polyphase waveform sets are summarized in Tab. 4 where $L$ is the transmitted code length and $M$ is the length of mismatched filter's coefficients. As shown in Tab. 4, the PSL of both BPSK and polyphase mismatched codes are improved significantly in comparison to the matched case. To measure the PSLR improvement of designed mismatched filters compared to the corresponding matched filters, we use a gain parameter defined as $G_{P S L R}=\left|P S L R_{\text {mismatched }}\right|-\left|P S L R_{\text {matched }}\right|$. For example for the BPSK code with $L=28$ and $M=220$, this gain is equal to $G_{P S L R}=51.58 \mathrm{~dB}$ and for polyphase code with $L=30$ and $M=200$, we have $G_{P S L R}=51.77 \mathrm{~dB}$. The interesting point is that the LPG in these cases has been kept as low as possible and is equal to $-0.72 \mathrm{~dB}$ and $-0.26 \mathrm{~dB}$, respectively. We study the performance 
obtained with the designed mismatched filter of the BPSK code with $L=28$ and $M=110$. Figure 4(a) shows the output of the matched filter as well as the output obtained for a mismatched filter computed with the proposed method with a constraint LPG of $-0.8 \mathrm{~dB}$. The PSLR for the matched filter is equal to $-22.92 \mathrm{~dB}$, while the PSLR for the mismatched filter is $-44.30 \mathrm{~dB}$, inducing a gain of $21.38 \mathrm{~dB}$. In addition, the measured LPG for the mismatched filter reaches to $-0.64 \mathrm{~dB}$. Fig. 4(b) presents the result for the same sequence by setting the mismatched code length to $M=220$. In this case, the PSLR for the mismatched filter is $-74.51 \mathrm{~dB}$, inducing a gain of $51.59 \mathrm{~dB}$. Also, the measured LPG is equal to $-0.72 \mathrm{~dB}$. The PSLR and ISLR measures versus Doppler shift with $L=28$ and $M=220$ are shown in Fig. 5 (a). Clearly, the ISLR of the designed mismatched filters is lower than that of the matched filter in the full Doppler frequency region. The PSLR of designed mismatched filters is also lower than that of matched filter in the full region, but it is more sensitive to the Doppler frequency shift than that of the matched filter. We evaluate the performance obtained with the designed mismatched filter of polyphase codes with $L=30$ and $M=110$. Figure 5 (b) shows the PSLR and ISLR metrics versus the Doppler shift for polyphase codes with $L=30$, and $M=200$. As it is clear, the ISLR of the designed mismatched filters is lower than that of the matched filter in the Doppler frequency region related to $f_{\mathrm{d}} \tau \in[-0.5,0.5]$. The PSLR of the designed mismatched filters is also lower than that of the matched filter in the full region, but compared to the matched filter, it is more sensitive to the Doppler frequency shift. Figure 6(a) shows the output of the matched filter as well as the output obtained for a mismatched filter computed with the proposed method with a constraint LPG of $-0.8 \mathrm{~dB}$. The matched filter PSLR is equal to $-29.05 \mathrm{~dB}$, while the mismatched filter PSLR is $-54.21 \mathrm{~dB}$, inducing a gain of $25.16 \mathrm{~dB}$. In addition, the measured LPG for the mismatched filter reaches to $-0.25 \mathrm{~dB}$. Figure 6(b) presents the result obtained for the same sequence by setting the mismatched code length to $M=200$. In this case, the PSLR for the mismatched filter is $-80.82 \mathrm{~dB}$, inducing a gain of $51.77 \mathrm{~dB}$. Also, the measured LPG is equal to $-0.26 \mathrm{~dB}$.
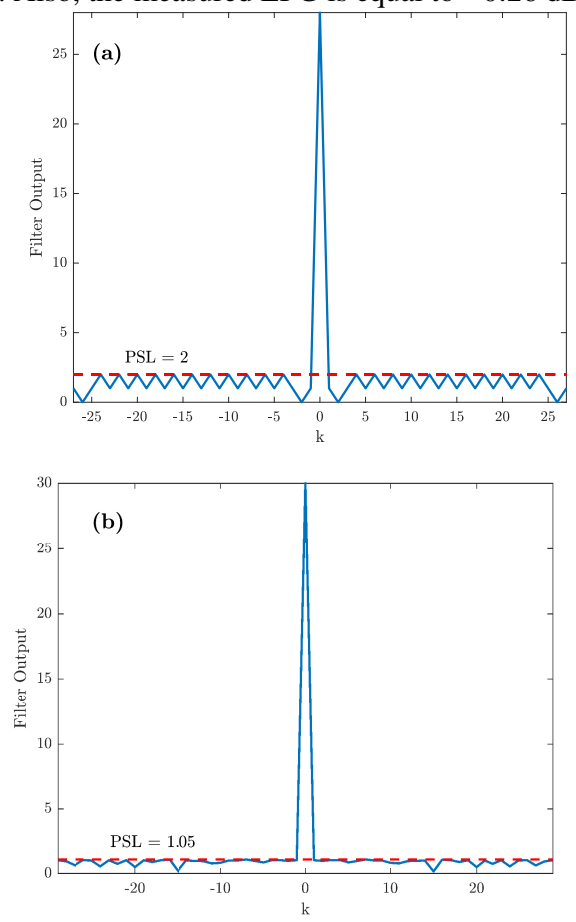

Fig. 3. The matched filter output (a) BPSK, $L=28$, and (b) Polyphase, $L=30$.

\begin{tabular}{|c|c|c|c|c|c|c|c|}
\hline Code & $L$ & $M$ & PSL & $\operatorname{PSLR}(\mathrm{dB})$ & LPG(dB) & ISLR(dB) & Gain(dB) \\
\hline \multirow{8}{*}{$\begin{array}{l}\frac{1}{\infty} \\
\frac{n}{n}\end{array}$} & \multirow{8}{*}{$\stackrel{\infty}{\sim}$} & 42 & 1.15 & -27.70 & -0.62 & -11.07 & 4.78 \\
\hline & & 56 & 0.90 & -29.83 & -0.74 & -11.95 & 6.91 \\
\hline & & 84 & 0.24 & -41.19 & -0.62 & -21.60 & 18.27 \\
\hline & & 110 & 0.17 & -44.30 & -0.64 & -23.74 & 21.38 \\
\hline & & 140 & 0.06 & -53.81 & -0.68 & -32.19 & 30.89 \\
\hline & & 168 & 0.02 & -62.72 & -0.71 & -40.26 & 39.79 \\
\hline & & 220 & $5.3 \times 10^{-4}$ & -74.51 & -0.72 & -50.95 & 51.59 \\
\hline & & 320 & $4.19 \times 10^{-4}$ & -96.49 & -0.72 & -71.47 & 73.57 \\
\hline \multirow{7}{*}{$\begin{array}{l}\frac{0}{0} \\
\text { 总 } \\
\frac{2}{2}\end{array}$} & \multirow{7}{*}{ p } & 50 & $\overline{0.53}$ & -35.06 & -0.28 & -16.65 & $\overline{6.01}$ \\
\hline & & 60 & 0.40 & -37.60 & -0.31 & -18.69 & 8.54 \\
\hline & & 90 & 0.08 & -51.03 & -0.24 & -30.71 & 21.97 \\
\hline & & 110 & 0.06 & -54.21 & -0.25 & -33.18 & 25.16 \\
\hline & & 150 & 0.01 & -67.50 & -0.26 & -45.28 & 38.44 \\
\hline & & 200 & $2.7 \times 10^{-5}$ & -80.82 & -0.26 & -57.47 & 51.77 \\
\hline & & 300 & $1.42 \times 10^{-4}$ & -106.48 & -0.27 & -82.10 & 77.43 \\
\hline
\end{tabular}

Tab. 4. PSL, PSLR, LPG, ISLR, and gain metrics of BPSK and polyphase codes with different values of $\boldsymbol{M}$.
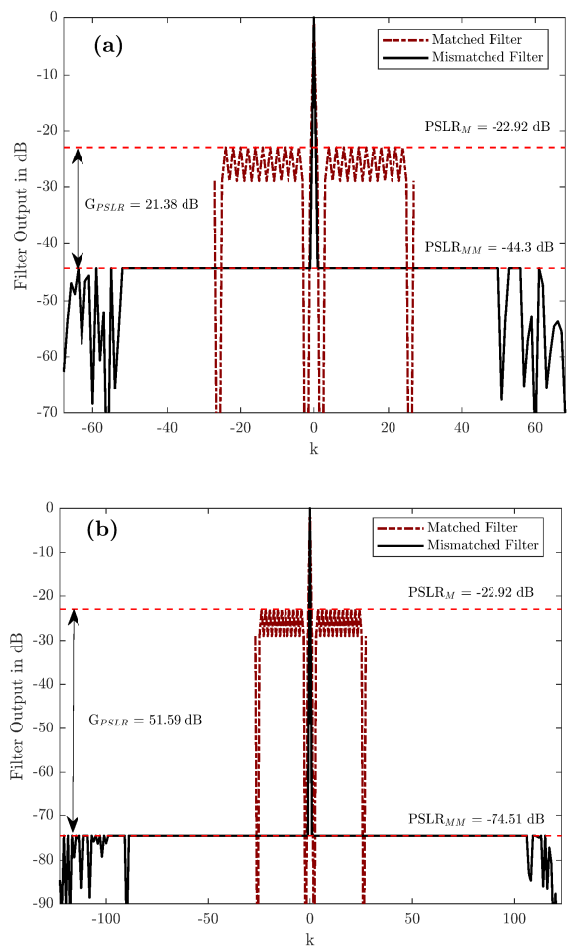

Fig. 4. Matched and mismatched filters for a BPSK code with $L=28$ and (a) $M=110$, (b) $M=220$.

To validate the simulation results, we implement one of the BPSK codes with the corresponding mismatched filter. In this experimental prototype, the real blocks used in a radar receiver are implemented in a virtex-4 FPGA, including analog to digital converter, Digital DownConversion (DDC), and pulse compression or (mis)matched filter blocks. The output of the pulse compression block is then analyzed. From Tab. 4, the BPSK code with $L=28$ and the corresponding mismatched filter with $M=110$ have been selected for implementation. Consider a radar system with the following specifications that works in normal mode, i.e., carrier frequency of $f_{\mathrm{c}}=200 \mathrm{MHz}$, intermediate frequency of $f_{\mathrm{IF}}=30$ $\mathrm{MHz}$, sampling frequency of $f_{\mathrm{c}}=24 \mathrm{MHz}$, and a chip duration of $T_{\text {chip }}=2 \mu \mathrm{s}$. In this case, there are 48 samples in each chip, which are downsampled with the rate of 48 in the DDC block.

Consider a target at a range of $R=50 \mathrm{~km}$ with a radar cross section of $\sigma=10 \mathrm{~m}^{2}$ that has a radial velocity of $v=100 \mathrm{~m} / \mathrm{s}$. According to the above assumptions and considering the noise power of $P_{\mathrm{n}}=-130 \mathrm{~dB}$, the output of the mismatched filter is shown in Fig. 7. 

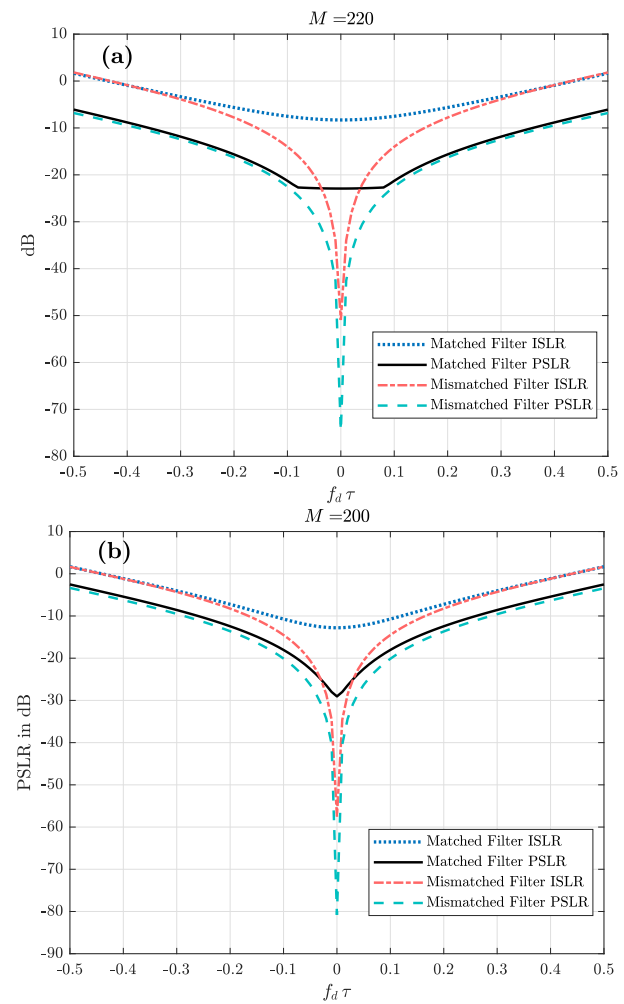

Fig. 5. PSLR and ISLR vs. Doppler shift for (a) BPSK code, $L=28, M=220$, (b) polyphase, $L=30, M=200$.
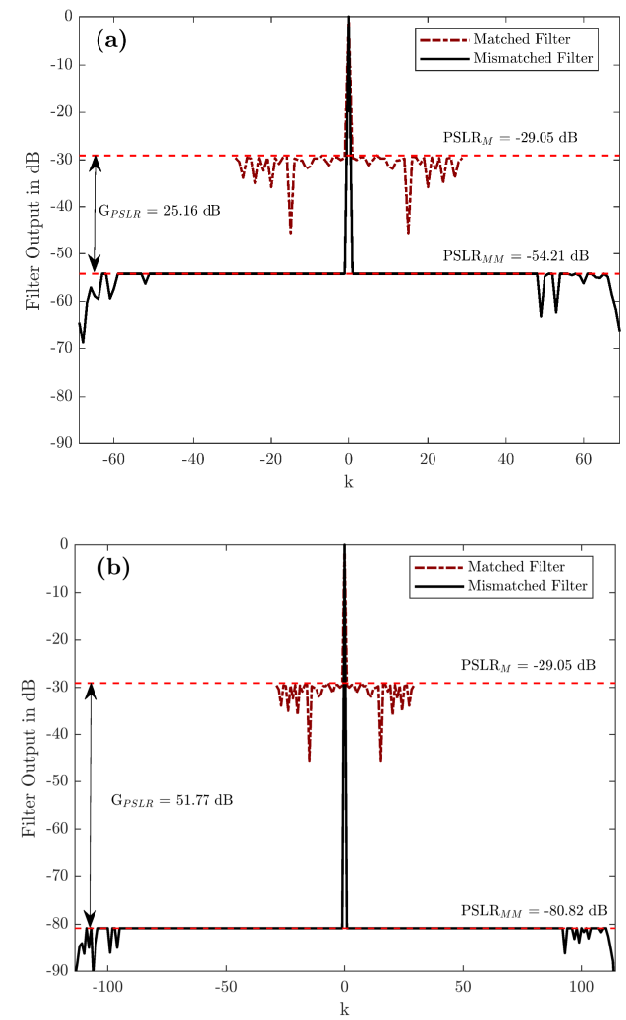

Fig. 6. Matched and mismatched filters outputs for polyphase code with $L=30$ and (a) $M=110$, (b) $M=200$.

It should be noted that if we remove the noise in this operation, the output of the pulse compression block will be nearly the same as the simulation result in Fig. 4(a). As can be seen in this figure, the PSLR in the case of additive noise is equal to
$-36.1 \mathrm{~dB}$ and in the case of no noise is equal to $-43.9 \mathrm{~dB}$ that is near to the simulation result of $-44.3 \mathrm{~dB}$. In the final step of our simulation, the mismatched filter design with Doppler constraints in the regions $f_{\mathrm{d}} \tau \in[-0.4,0.4]$ and $f_{\mathrm{d}} \tau \in[-0.2,0.2]$ is applied for BPSK and polyphase codes, respectively. The matched filter, the designed mismatched filter with no Doppler constraints, and the designed mismatched filter with Doppler constraints are then used to process the signals with different Doppler shifts in accordance with $f_{\mathrm{d}} \tau \in[-0.5,0.5]$. Figures 8(a) and 8(b) illustrate the PSLR in terms of the Doppler shift for BPSK and polyphase codes, respectively. As expected, we can observe that the mismatched filter with no Doppler constraints is not very robust to the Doppler shift, i.e., its PSLR rapidly increases with the target radial velocity and becomes even almost worst than the PSLR of the matched filter in the regions $\left|f_{\mathrm{d}} \tau\right|>0.15$ and $\left|f_{\mathrm{d}} \tau\right|>0.05$ for BPSK and polyphase codes, respectively. In contrast, the PSLR provided by the designed mismatched filter with Doppler constraints remains relatively flat in the regions $f_{\mathrm{d}} \tau \in[-0.4,0.4]$ for BPSK and $f_{\mathrm{d}} \tau \in[-0.2,0.2]$ for polyphase code, and better than the matched filter in the region $\left|f_{\mathrm{d}} \tau\right|>0.15$ for BPSK and the whole Doppler range for polyphase code as well.

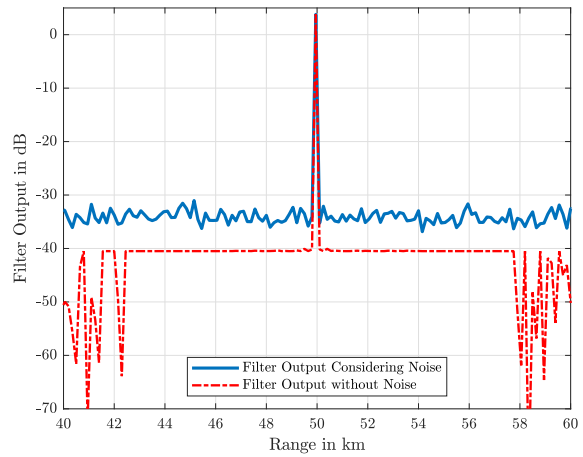

Fig. 7. Experimental results of the mismatched filter output, $L=28, M=110, R=50 \mathrm{~km}$, with and without noise.
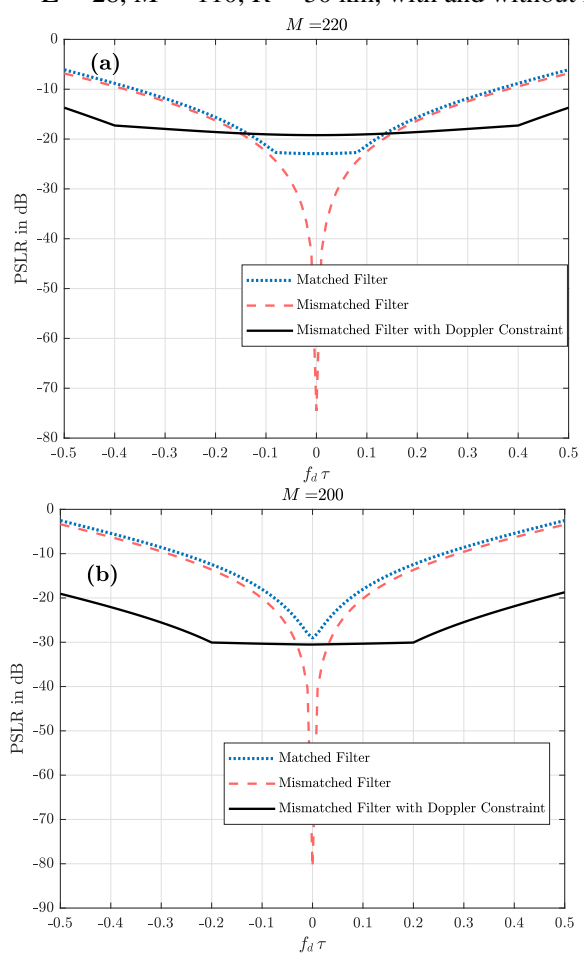

Fig. 8. PSLR vs. Doppler shift for (a) BPSK code, $L=28$, $M=220$, (b) polyphase codes, $L=30, M=200$. 


\section{Conclusion}

In this paper, we proposed two algorithms for designing matched and mismatched filters taking the PSL and LPG criteria into account. The matched filter design algorithm employed the least- $\mathrm{p}^{\text {th }}$ norm minimax based on the genetic algorithm, while the mismatched design method was based on the semidefinite programming. The results of the matched filter design showed the satisfactory PSLR for BPSK and polyphase codes. The performance of the mismatched filter design was evaluated through the simulations, and great improvement in the PSLR were obtained and the LPG was negligible during the design procedure. For the mismatched filter design with Doppler constraints, our simulation results demonstrated that although the classic and designed mismatched filters are not robust to Doppler shifts, such Doppler constraints extensively improve the robustness of PSLR for a predefined Doppler region.

\section{References}

[1] MIDDLETON, D. Non-Gaussian Statistical Communication Theory. 1st ed. New York (USA): John Wiley and Sons, 2012. ISBN: 978-1-118-16195-1

[2] CHITGARHA, M. M., RADMARD, M., MAJD, M. N., et al. MIMO radar signal design to improve the MIMO ambiguity function via maximizing its peak. ELSEVIER Signal Processing, 2016, vol. 118 , p. 139-152. DOI: 10.1016/j.sigpro.2015.06.024

[3] RICHARDS, M. A. Fundamentals of Radar Signal Processing. 2nd ed. New York (USA): McGraw-Hill, 2014. ISBN: 9780071798327

[4] LEVANON, N., MOZESON, E. Radar Signals. 1st ed. Hoboken (USA): John Wiley and Sons, 2004. ISBN 0-471-47378-2

[5] CHANG, G., YU, X., YU, C. Discrete frequency and phase coding waveform for MIMO radar. Radioengineering, 2017, vol. 26, no. 3 , p. 835-841. DOI: 10.13164/re.2017.0835

[6] WANG, Z., LIAO, G., YANG, Z. Space-frequency modulation radarcommunication and mismatched filtering. IEEE Access, 2018, vol. 6, p. 24837-24845. DOI: 10.1109/ACCESS.2018.2829731

[7] XU, L., ZHOU, S., LIU, H., et al. Distributed multipleinput-multiple-output radar waveform and mismatched filter design with expanded mainlobe. In 2016 CIE International Conference on Radar (RADAR). Guangzhou (China), 2016, p. 1-5. DOI: $10.1109 /$ RADAR.2016.8059149

[8] WANG, H., LI, W., WANG, H., et al. Radar waveform strategy based on game theory. Radioengineering, 2019, vol. 28, no. 4, p. 757-764. DOI: $10.13164 /$ re.2019.0757

[9] BADEN, J., COHEN, M. Optimal peak sidelobe filters for biphase pulse compression. In Proceedings of IEEE International Radar Conference. Arlington (USA), 1990, p. 249-252. DOI: 10.1109/RADAR.1990.201171

[10] SAHOO, A. K., PANDA, G., PRADHAN, P. M. Efficient design of pulse compression codes using multi objective genetic algorithm. In Proceedings of World Congress on Nature \& Biologically Inspired Computing. Coimbatore (India), 2009, p. 324-329. DOI: $10.1109 / \mathrm{NABIC} .2009 .5393731$

[11] BADEN, J., DAVIS, M. S., SCHMIEDER, L. Efficient energy gradient calculations for binary and polyphase sequences. In Proceedings of IEEE Radar Conference. Arlington (USA), 2015, p. 304-309. DOI: 10.1109/RADAR.2015.7131014
[12] AITTOMAKI, T., KOIVUNEN, V. Mismatched filter design and interference mitigation for MIMO radars. IEEE Transactions on Signal Processing, 2017, vol. 65, no. 2, p. 454-466. DOI: 10.1109/TSP.2016.2620960

[13] BADEN, J. M., O’DONNELL, B., SCHMIEDER, L. Multi objective sequence design via gradient descent methods. IEEE Transactions on Aerospace and Electronic Systems, 2018, vol. 54, no. 3, p. 1237-1252. DOI: 10.1109/TAES.2017.2780538

[14] YU, G., LIANG, J., LI, J., et al. A. Sequence set design with accurately controlled correlation properties. IEEE Transactions on Aerospace and Electronic Systems, 2018, vol. 54, no. 6, p. 3032-3046. DOI: 10.1109/TAES.2018.2836778

[15] ALAIE, M. B., OLAMAEI, S. A. Waveform design for TDM-MIMO radar systems. ELSEVIER Signal Processing, 2020, vol. 167, p. 1-8. DOI: 10.1016/j.sigpro.2019.107307

[16] FAN, W., LIANG, J., YU, G., et al. Minimum local peak sidelobe level waveform design with correlation and/or spectral constraints. ELSEVIER Signal Processing, 2020, vol. 171, p. 2-8. DOI: $10.1016 /$ j.sigpro.2019.107450

[17] SONG, X., ZHOU, S., WILLETT, P. Reducing the waveform cross correlation of MIMO radar with space time coding. IEEE Transactions on Signal Processing, 2010, vol. 58, no. 8, p. 4213-4224. DOI: 10.1109/TSP.2010.2048207

[18] BOYD, S., VANDENBERGHE, L. Convex Optimization. 1st ed. Cambridge (UK): Cambridge University Press, 2004. ISBN: 978-0-521-83378-3

[19] LOFBERG, J. YALMIP: A toolbox for modeling and optimization in Matlab. In Proceedings of IEEE International Conference on Robotics and Automation. Taipei (Taiwan), 2004. DOI: $10.1109 /$ CACSD.2004.1393890

[20] LIN, R., SOLTANALIAN, M., TANG, B., et al. Efficient design of binary sequences with low autocorrelation sidelobes. IEEE Transactions on Signal Processing, 2019, vol. 67, no. 24, p. 6397-6410. DOI: $10.1109 /$ TSP.2019.2954525

[21] STOICA, P., HE, H., LI, J. New algorithms for designing unimodular sequences with good correlation properties. IEEE Transactions on Signal Processing, 2009, vol. 57, no. 4, p. 1415-1425. DOI: 10.1109/TSP.2009.2012562

\section{About the Authors ...}

Mohammad Mehdi PISHROW received the M.Sc. degree in Communication Engineering from Shiraz University, Iran, in 2011. $\mathrm{He}$ is currently pursuing the Ph.D. degree in Communication Engineering at the Department of Electrical Engineering, Yazd University, Iran. His research interests are related to waveform design for active sensing, optimization theory applied to radar signal processing, and statistical/array signal processing.

Jamshid ABOUEI (corresponding author) received the Ph.D. degree in Electrical Engineering from University of Waterloo, Canada, in 2009. He was promoted to Associate Professor at the Department of Electrical Engineering, Yazd University, Iran, in 2015. Currently, Dr. Abouei directs the research group at the Wireless Networking Laboratory (WINEL), Yazd University, Iran.

Hesam GHAFERI received the M.Sc. degree in Electrical Engineering from Islamic Azad University, Sepidan, Iran, in 2014. His research interests include digital signal processing, optimization theory, and implementation of signal processing based on FPGA. 